\title{
An EOQ model for deteriorating items analyzing retailer's optimal strategy under trade credit and return policy with nonlinear demand and resalable returns
}

\author{
Mamta Kumari and Pijus Kanti De* \\ Department of Mathematics, National Institute of Technology Silchar, Silchar -788010, India \\ mamta_rs@math.nits.ac.in,pkde@math.nits.ac.in
}

\begin{tabular}{|c|c|}
\hline ARTICLE INFO & ABSTRACT \\
\hline $\begin{array}{l}\text { Article history: } \\
\text { Received: } 30 \text { September } 2020 \\
\text { Accepted: } 1 \text { December } 2021 \\
\text { Available Online: } 2 \text { January } 2022\end{array}$ & \multirow{3}{*}{$\begin{array}{l}\text { This paper presents an EOQ model where demand is dependent upon time and } \\
\text { selling price. In the proposed model of inventory, the retailer allows its } \\
\text { unsatisfied customers to return their product whereas the manufacturer offers a } \\
\text { full trade credit policy to the retailer. To make our model realistic, we have } \\
\text { assumed that the product returned can be resold with the same selling price. } \\
\text { Number of returns is a function of demand. In this proposed inventory model } \\
\text { considering deterioration, the retailer does not fully reimburse its customers for } \\
\text { the returned product. The primary purpose of this inventory model is to } \\
\text { determine the optimal selling price, optimal order quantity, and optimal } \\
\text { replenishment cycle length in order to maximize the retailer's total profit earned } \\
\text { per unit time. A numerical example is also presented and a sensitivity analysis is } \\
\text { carried to highlight the findings of the suggested inventory model. }\end{array}$} \\
\hline $\begin{array}{l}\text { Keywords: } \\
\text { Inventory } \\
\text { Trade credit policy } \\
\text { Resalable returns } \\
\text { Deteriorating item } \\
\text { Refund }\end{array}$ & \\
\hline $\begin{array}{l}\text { sification 2010: } \\
306\end{array}$ & \\
\hline
\end{tabular}

\section{Introduction}

Return policies are offered as an attractiveness pill to drag customers by the seller. The return policy allows consumers who are unsatisfied with their purchase to return the product and get a refund either in terms of money or some gift vouchers as stated by the company in its policies. In today's competitive market, many companies offer return policies to their consumers in order to increase sales. Bechwati and Siegel [1] concluded from their research that customers consider return policy as a signal for purchasing products from a store.

Owing to the return policies, customer returns are growing drastically in the past few decades since customers are taking advantage of their strength which can be understood by the retail industry slogan that "consumers are king". Gentry [2] concluded that the rate of product return can be as high as $35 \%$ of the initial stock. Mostard et al. [3] observed that return rate can be larger than $18 \%$ in the case of mail orders and it can even reach to $74 \%$ for fashion products. Due to the high return rate, it becomes difficult for the retailer to handle its inventory and associated cost. Palmquist [4] concluded that product returns cost US

\footnotetext{
${ }^{*}$ Corresponding author
}

retailers and manufacturers approximately $100 \$$ billion annually in reverse logistics.

Many research works have been done in the past decades to analyze whether offering a return policy is beneficial to the seller or not. Recent research recommends to follow a restrictive return policy in order to reduce consumer abuse of leniency of return policies, manage reverse logistic costs and increase profitability [5-8]. Peterson and Kumar [9] studied whether product return is a necessary evil? It's antecedent and consequences. Then, Janakiraman et al. [10] examined the effect of leniency of return policies on consumer behavior towards return and purchase decisions by conducting a review of 21 papers. After that, Khouja et. al. [11] studied retailer's performance under the effect of price adjustment policies and returns. Many research works have also appeared in the literature on deteriorating items with the return policy. Wang et al. [12] formulated an EOQ model with resalable returns for deteriorating items. Ghoreishi et al. [13] framed an EOQ model under inflation to determine the pricing and ordering strategy of deteriorating items under customer returns. Similarly, many relevant research work has been done on EOQ model considering different situations [14- 
17]. Deterioration refers to decay, evaporation, spoilage, decay, and loss of utility of the product. In full trade credit policy, buyer does not need to pay immediately upon receipt of merchandise and has the opportunity to settle the amount at the end of the delay time. If he fails to pay the money at the end of credit time, then he is bound to pay interest on the unpaid amount.

If we have a glance at the literature, plenty of works have been done on deteriorating items employing trade credit policy under different circumstances [1819]. However, until now, no research work has been done in the past, which jointly analyzes the effect of return and trade credit terms on deteriorating items with resalable returns. There is a research gap lying on jointly studying the impact of both return policy and trade credit policy in the business of management of inventory. In this paper, according to the best of author knowledge, first attempt has been made to fill this research gap by jointly analyzing the impact of both trade credit and return policy in the supply chain that contributes to the past literature. Both trade credit policy and return policy importance is growing day by day in the supply chain, so it becomes necessary to analyze the impact of jointly considering the aforementioned policies on the pricing and inventory decisions in the supply chain.

Specifically, this paper studies a manufacturer retailer - customer business relationship with the following features: (a) retailer is offered a full trade credit period by the manufacturer, (b) retailer also offers a return policy to its buyer to generate sales, (c) demand is dependent upon selling price as well as time, (d) deterioration occurs, and (e) rate of return is observed to be dependent upon selling price as well as time. This research work intends to determine the optimal replenishment cycle length and the optimal selling price in order to maximize the total profit earned by the retailer per unit time. A numerical example is also demonstrated to highlight the findings and a sensitivity analysis is carried out to discuss the results.

Further, this paper is organized as follows: Section 2 demonstrates the notations and assumptions used in order to establish the EOQ model mathematically. Thereafter, Section 3 develops the mathematical model considering demand, return and trade credit policy. Section 4 illustrates a numerical example to highlight the findings. Finally, Section 5 depicts a sensitivity analysis and highlights some results. Lastly, Section 6 ends up with few remarkable conclusions and future research directions.

\section{Assumptions and notations}

\subsection{Notations}

Table 1. Notations that are used in formulating the inventory model

\begin{tabular}{|c|c|c|c|c|c|}
\hline Notation & Units & Description & Notation & Units & Description \\
\hline $\bar{O}$ & \$/order & ordering cost per order & $\overline{I_{e}}$ & $\%$ /unit & interest earned by the \\
\hline$c$ & \$/unit & purchase price per unit & & time & retailer \\
\hline$h$ & $\begin{array}{l}\text { \$/unit/unit } \\
\text { time }\end{array}$ & $\begin{array}{l}\text { holding cost per unit item } \\
\text { per unit time }\end{array}$ & $I_{p}$ & $\begin{array}{l}\% / \text { unit } \\
\text { time }\end{array}$ & $\begin{array}{l}\text { interest paid by the } \\
\text { retailer to the manufacturer }\end{array}$ \\
\hline$\theta$ & & $\begin{array}{l}\text { constant deterioration } \\
\text { rate, } 0<\theta<1\end{array}$ & Decision v & variables: & \\
\hline$M$ & unit time & $\begin{array}{l}\text { trade credit period } \\
\text { offered by the manufacturer } \\
\text { to the retailer }\end{array}$ & $\begin{array}{l}p \\
T\end{array}$ & $\begin{array}{l}\text { \$/unit } \\
\text { unit time }\end{array}$ & $\begin{array}{l}\text { selling price per unit } \\
\text { time at which inventory } \\
\text { level reaches to zero }\end{array}$ \\
\hline$t_{1}$ & unit time & $\begin{array}{l}\text { time at which deterioration } \\
\text { starts }\end{array}$ & $T P(p, T)$ & $\begin{array}{l}\text { \$/unit } \\
\text { time }\end{array}$ & total profit per unit time \\
\hline
\end{tabular}

\subsection{Assumptions}

1. Planning horizon of the inventory system is assumed to be infinite.

2. Single item with non-instantaneous deterioration is considered.

3. Instantaneous replenishment rate, where lead-time is considered negligible.

4. Shortages are not permitted.

5. Demand rate is given by:

$$
D(p, t)=(a-b p) e^{\lambda t} \text { where } a>0, b>0
$$

It is a linearly decreasing function of price and increases (decreases) exponentially with time as $\lambda>0(\lambda<0)$.

6. A full trade credit policy is offered by the manufacturer to the retailer.

7. It is assumed that customer return increases with the goods sold. So,

$$
R(p, t)=\alpha D(p, t) \quad \text { where } 0 \leq \alpha<1
$$

Customers are allowed to return the product during any phase of the length of the replenishment cycle. Products returned can be resold at the same selling price. The retailer does not return the full amount to its customers for the returned goods. He just offers 
$50 \%$ of the initial amount of the product.

\section{Model formulation}

This section derives, in detail, an inventory model with trade credit and return policy. Initially, $\boldsymbol{Q}$ units of goods exist in the inventory model. During the time interval from $\mathbf{0}$ to $\boldsymbol{t}_{\mathbf{1}}$, no deterioration takes place. During this time, the level of inventory changes due to returns and demand. From time $\mathrm{t}=\boldsymbol{t}_{\mathbf{1}}$, deterioration of items at the rate $\boldsymbol{\theta}$ starts taking place. From time $\boldsymbol{t}_{\mathbf{1}}$ to $\boldsymbol{T}$, inventory level changes because of returns, deterioration and demand. At time $\mathrm{t}=\boldsymbol{T}$, the level of inventory demises to zero. Thereafter, a replenishment order of $\boldsymbol{Q}$ units is placed. The change in inventory level with the passage of time is shown in Figure 1. The arrival of the ordered goods marks the beginning of the next cycle. The retailer is offered a trade credit period of $\boldsymbol{M}$ to settle the account by the manufacturer.

The retailer also allows its customers to return products, during any time of the replenishment cycle with a condition. The condition is that the retailer will refund only $50 \%$ of the initial amount of the product. The inventory situation is best governed by the following differential equations:

$$
\begin{aligned}
& \frac{d I}{d t}=-(a-b p) e^{\lambda t}+\alpha(a-b p) e^{\lambda t}, \quad 0 \leq t \leq t_{1} \\
& \frac{d I}{d t}=-(a-b p) e^{\lambda t}+\alpha(a-b p) e^{\lambda t}-\theta I(t) \\
& t_{1} \leq t \leq T
\end{aligned}
$$

with the boundary conditions $I(0)=Q$ and $I(T)=0$.

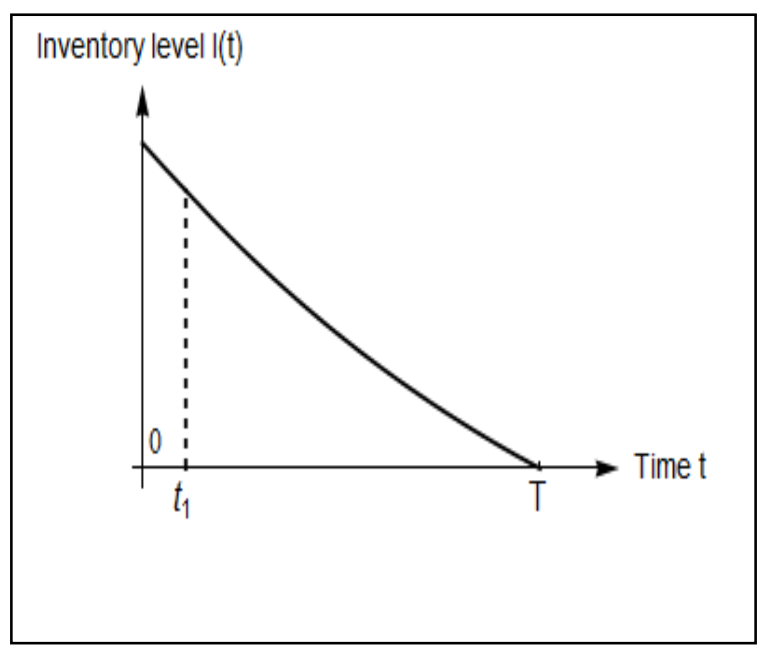

Figure 1. Inventory level at any time $t$

Solving the differential equations (1) and (2) along with the above-mentioned boundary conditions we get,

$$
\begin{aligned}
& I(t)=Q+\frac{(\alpha-1)(a-b p)\left(e^{\lambda t}-1\right)}{\lambda}, \quad 0 \leq t \leq t_{1} \\
& I(t)=\frac{(a-b p)(\alpha-1) e^{-\theta t}}{(\lambda+\theta)}\left[e^{(\lambda+\theta) t}-e^{(\lambda+\theta) T}\right] \\
& t_{1} \leq t \leq T
\end{aligned}
$$

Using the continuity condition from the equation (3) and (4) at the point $t=t_{1}$, we get

$$
\begin{aligned}
Q= & {\left[\frac{(a-b p)(\alpha-1) e^{-\theta t_{1}}}{(\lambda+\theta)}\left[e^{(\lambda+\theta) t_{1}}-e^{(\lambda+\theta) T}\right]\right]-} \\
& {\left[\frac{(\alpha-1)(a-b p)\left(e^{\left.\lambda t_{1}-1\right)}\right.}{\lambda}\right] }
\end{aligned}
$$

Various costs associated with this model are defined as follows:

1. Ordering cost $=o$

2. Purchasing cost $=c Q$

3. Sales revenue collected over the cycle (SR)

$$
\begin{aligned}
& =p\left[\int_{0}^{T} D(p, t) d t-\int_{0}^{T} \frac{R(p, t)}{2} d t\right] \\
& =p\left[\int_{0}^{T}(a-b p) e^{\lambda t} d t-\int_{0}^{T} \frac{\alpha(a-b p) e^{\lambda t}}{2} d t\right] \\
& =\left[\frac{p(a-b p)\left(e^{\lambda T}-1\right)}{\lambda}\right]\left[1-\frac{\alpha}{2}\right]
\end{aligned}
$$

4. Deterioration cost over the cycle (DC)

$$
\begin{aligned}
& =c \int_{t_{1}}^{T} \theta I(t) d t \\
& =c \int_{t_{1}}^{T} \theta\left[\frac{(a-b p)(\alpha-1) e^{-\theta t}}{(\lambda+\theta)}\right]\left[e^{(\lambda+\theta) t}-\right. \\
& \left.e^{(\lambda+\theta) T}\right] d t \\
& \left.\left.\left.\theta e^{\lambda t_{1}}-\lambda e^{(\lambda+\theta) T} e^{-\theta t_{1}}\right]\right] /[\lambda(\lambda+\theta)]\right]
\end{aligned}
$$

5. Holding cost over the cycle $(\mathrm{HC})$

$$
\begin{aligned}
=h & {\left[\int_{0}^{t_{1}} I(t) d t+\int_{t_{1}}^{T} I(t) d t\right] } \\
=h & {\left[\int_{0}^{t_{1}}\left[Q+\frac{(\alpha-1)(a-b p)\left(e^{\lambda t}-1\right)}{\lambda}\right] d t+\right.} \\
& \int_{t_{1}}^{T}\left[\frac { ( a - b p ) ( \alpha - 1 ) e ^ { - \theta t } } { ( \lambda + \theta ) } \left[e^{(\lambda+\theta) t}-\right.\right. \\
& \left.\left.e^{(\lambda+\theta) T]}\right] d t\right] \\
= & h\left[Q t_{1}+\left[\frac{(\alpha-1)(a-b p)\left(e^{\left.\lambda t_{1}-\lambda t_{1}-1\right)}\right.}{\lambda^{2}}\right]-\right. \\
& {\left[\frac{(a-b p)(\alpha-1)}{\theta \lambda(\theta+\lambda)}\right]\left[\theta e^{\lambda t_{1}-(\theta+\lambda) e^{\lambda T}+}\right.} \\
& \left.\lambda e^{(\theta+\lambda) T} e^{-\theta t_{1}}\right]
\end{aligned}
$$

6. Trade credit:

Manufacturer offers the retailer a delay period of $M$. According to the values of $M, t_{1}$ and $T$ three subcases arise:
(1) $0 \leq M \leq t_{1}$
(2) $t_{1} \leq M \leq T$
(3) $M \geq T$

6.1. $0 \leq M \leq t_{1}$ 
In this case, the credit period offered by the manufacturer to the retailer is less than the time at which deterioration starts in the inventory. After the end of credit period $M$, the retailer is subject to interest charges and needs to pay interest during the time interval $[M, T]$. Therefore, interest paid is calculated as follows:

$$
\begin{aligned}
\mathrm{IP}= & c I_{p}\left[\int_{M}^{t_{1}} I(t) d t+\int_{t_{1}}^{T} I(t) d t\right] \\
= & c I_{p}\left[\int_{M}^{t_{1}}\left[Q+\frac{(\alpha-1)(a-b p)\left(e^{\lambda t}-1\right)}{\lambda}\right] d t+\right. \\
& \int_{t_{1}}^{T}\left[\frac { ( a - b p ) ( \alpha - 1 ) e ^ { - \theta t } } { ( \lambda + \theta ) } \left[e^{(\lambda+\theta) t}-\right.\right. \\
& \left.\left.\left.e^{(\lambda+\theta) T}\right]\right] d t\right] \\
= & c I_{p}\left[Q\left(t_{1}-M\right)+\left[\frac{(\alpha-1)(a-b p)}{\lambda}\right][M-\right. \\
& \left.t_{1}+\left(\left(e^{\lambda t_{1}}-e^{\lambda M}\right) / \lambda\right)\right]+ \\
& {[(\alpha-1)(a-b p) /(\lambda+\theta)]\left[\frac{e^{\lambda T}}{\theta \lambda}(\theta+\right.} \\
& \left.\left.\lambda)-\frac{e^{\lambda t_{1}}}{\lambda}-\frac{e^{(\lambda+\theta) T} e^{-\theta t_{1}}}{\theta}\right]\right]
\end{aligned}
$$

The retailer also earns interest from time period 0 to $M$. It is calculated as follows:

$$
\begin{aligned}
\mathrm{IE}= & p I_{e}\left[\int_{0}^{M} \int_{0}^{t}(a-b p) e^{\lambda u} d u d t-\right. \\
& \left.\int_{0}^{M} \int_{0}^{t} \frac{\alpha(a-b p) e^{\lambda u}}{2} d u d t\right] \\
= & p I_{e}\left[\frac{(a-b p)}{\lambda^{2}}\right]\left[e^{\lambda M}-\lambda M-1\right]\left[1-\frac{\alpha}{2}\right]
\end{aligned}
$$

The total profit per unit time is calculated as follows:

$$
T P_{1}(p, T)=\frac{S R+I E-o-I P-H C-D C-P C}{T}
$$

Therefore,

$$
\begin{aligned}
& T P_{1}(p, T)=[1 / T]\left[\frac{p(a-b p)\left(e^{\lambda T}-1\right)}{\lambda}\right]\left[1-\frac{\alpha}{2}\right]+ \\
& p I_{e}\left[\frac{(a-b p)}{\lambda^{2}}\right]\left[e^{\lambda M}-\lambda M-1\right]\left[1-\frac{\alpha}{2}\right]-o- \\
& c I_{p}\left[Q\left(t_{1}-M\right)+\left[\frac{(\alpha-1)(a-b p)}{\lambda}\right]\left[M-t_{1}+\right.\right. \\
& \left.\left(\frac{e^{\lambda t_{1}-e^{\lambda M}}}{\lambda}\right)\right]+\left[\frac{(\alpha-1)(a-b p)}{\lambda+\theta}\right]\left[\frac{e^{\lambda T}}{\theta \lambda}(\theta+\lambda)-\right. \\
& \left.\frac{e^{\lambda t_{1}}}{\lambda}-\frac{e^{(\lambda+\theta) T} e^{-\theta t_{1}}}{\theta}\right]-h\left[Q t_{1}+\right. \\
& {\left[\frac{(\alpha-1)(a-b p)\left(e^{\left.\lambda t_{1}-\lambda t_{1}-1\right)}\right.}{\lambda^{2}}\right]-} \\
& {\left[\frac{(a-b p)(\alpha-1)}{\theta \lambda(\theta+\lambda)}\right]\left[\theta e^{\lambda t_{1}}+\lambda e^{(\lambda+\theta) T} e^{-\theta t_{1}}-\right.} \\
& \left.\left.(\theta+\lambda) e^{\lambda T}\right]\right]-
\end{aligned}
$$

$$
\begin{aligned}
& {\left[\frac{c(a-b p)(\alpha-1)\left[(\theta+\lambda) e^{\lambda T}-\theta e^{\lambda t_{1}}-\lambda e^{(\lambda+\theta) T} e^{-\theta t_{1}}\right]}{[\lambda(\lambda+\theta)]}\right]-} \\
& c\left[\left[\frac{(a-b p)(\alpha-1) e^{-\theta t_{1}}}{(\lambda+\theta)}\left[e^{(\lambda+\theta) t_{1}}-e^{(\lambda+\theta) T}\right]\right]-\right. \\
& \left.\left[\frac{(\alpha-1)(a-b p)\left(e^{\left.\lambda t_{1}-1\right)}\right.}{\lambda}\right]\right]
\end{aligned}
$$

Problem - 1:

Maximize $T P_{1}(p, T)=\frac{W_{1}}{T}$

where $W_{1}=S R+I E-o-I P-H C-D C-P C$

subject to $0 \leq M \leq t_{1}$

\section{2. $t_{1} \leq M \leq T$}

In this subcase, the credit period offered to the retailer by the manufacturer is greater than the time at which deterioration starts and less than the time by which the inventory level reaches zero. After the end of credit period $M$, the retailer is subject to interest charges and needs to pay interest during the time interval $[M, T]$. It is calculated as follows:

$$
\begin{aligned}
\mathrm{IP}= & c I_{p}\left[\int_{M}^{T} I(t) d t\right] \\
= & c I_{p}\left[\int _ { M } ^ { T } \left[\frac { ( a - b p ) ( \alpha - 1 ) e ^ { - \theta t } } { ( \lambda + \theta ) } \left[e^{(\lambda+\theta) t}-\right.\right.\right. \\
& \left.\left.e^{(\lambda+\theta) T}\right] d t\right] \\
= & c I_{P}[[(\alpha-1)(a-b p) /(\lambda+ \\
& \left.\theta)]\left[\frac{e^{\lambda T}}{\theta \lambda}(\theta+\lambda)-\frac{e^{\lambda M}}{\lambda}-\frac{e^{(\lambda+\theta) T} e^{-\theta M}}{\theta}\right]\right]
\end{aligned}
$$

The retailer also earns interest from time period 0 to $M$. It is calculated as follows:

$$
\begin{aligned}
\mathrm{IE}= & {\left[\int_{0}^{M} \int_{0}^{t}(a-b p) e^{\lambda u} d u d t-\right.} \\
& \left.\int_{0}^{M} \int_{0}^{t} \frac{\alpha(a-b p) e^{\lambda u}}{2} d u d t\right] \\
= & p I_{e}\left[\frac{(a-b p)}{\lambda^{2}}\right]\left[e^{\lambda M}-\lambda M-1\right]\left[1-\frac{\alpha}{2}\right]
\end{aligned}
$$

The total profit per unit time is calculated as follows:

$$
T P_{2}(p, T)=\frac{S R+I E-o-I P-H C-D C-P C}{T}
$$

Therefore,

$$
\begin{aligned}
& T P_{2}(p, T)=[1 / T]\left[\left[\frac{p(a-b p)\left(e^{\lambda T}-1\right)}{\lambda}\right]\left[1-\frac{\alpha}{2}\right]+\right. \\
& p I_{e}\left[\frac{(a-b p)}{\lambda^{2}}\right]\left[e^{\lambda M}-\lambda M-1\right]\left[1-\frac{\alpha}{2}\right]-o- \\
& c I_{P}\left[[ \frac { ( \alpha - 1 ) ( a - b p ) } { \lambda + \theta } ] \left[\frac{e^{\lambda T}}{\theta \lambda}(\theta+\lambda)-\frac{e^{\lambda M}}{\lambda}-\right.\right.
\end{aligned}
$$




$$
\begin{aligned}
& \left.\left.\frac{e^{(\lambda+\theta) T} e^{-\theta M}}{\theta}\right]\right]-h\left[Q t_{1}+\right. \\
& {\left[\frac{(\alpha-1)(a-b p)\left(e^{\left.\lambda t_{1}-\lambda t_{1}-1\right)}\right.}{\lambda^{2}}\right]-} \\
& {\left[\frac{(a-b p)(\alpha-1)}{\theta \lambda(\theta+\lambda)}\right]\left[\theta e^{\lambda t_{1}}+\lambda e^{(\lambda+\theta) T} e^{-\theta t_{1}}-\right.} \\
& \left.\left.(\theta+\lambda) e^{\lambda T}\right]\right]- \\
& {\left[\frac{c(a-b p)(\alpha-1)\left[(\theta+\lambda) e^{\lambda T}-\theta e^{\left.\lambda t_{1}-\lambda e^{(\lambda+\theta) T} e^{-\theta t_{1}}\right]}\right.}{[\lambda(\lambda+\theta)]}\right]-} \\
& C\left[\frac{(a-b p)(\alpha-1) e^{-\theta t_{1}}}{(\lambda+\theta)}\left[e^{(\lambda+\theta) t_{1}}-e^{(\lambda+\theta) T}\right]\right]- \\
& \left.\left[\frac{(\alpha-1)(a-b p)\left(e^{\left.\lambda t_{1}-1\right)}\right.}{\lambda}\right]\right]
\end{aligned}
$$

\section{Problem 2:}

Maximize $T P_{2}(p, T)=\frac{W_{2}}{T}$

where $W_{2}=S R+I E-o-I P-H C-D C-P C$ subject to $t_{1} \leq M \leq T$

\section{3. $M \geq T$}

In this subcase, the delay period offered by the retailer to the manufacturer is greater than the time by which level of inventory drops down to zero. By this time the retailer clears all his debt. Hence,

$$
\text { IP }=0
$$

The retailer earns interest from time period 0 to $M$. It is calculated as follows:

$$
\begin{aligned}
\mathrm{IE}= & p I_{e}\left[\int_{0}^{T} \int_{0}^{t}(a-b p) e^{\lambda u} d u d t-\right. \\
& \int_{0}^{T} \int_{0}^{t} \frac{\alpha(a-b p) e^{\lambda u}}{2} d u d t+(M-T) \int_{0}^{T}(a- \\
& \left.b p) e^{\lambda t} d t-(M-T) \int_{0}^{T} \frac{\alpha(a-b p) e^{\lambda t}}{2} d t\right] \\
= & p I_{e}\left[\left[\frac{(a-b p)(M-T)\left(e^{\lambda T}-1\right)}{\lambda}\right]\left[1-\frac{\alpha}{2}\right]+\right. \\
& {\left.\left[\frac{(a-b p)\left(e^{\lambda T}-T \lambda-1\right)}{\lambda^{2}}\right]\left[1-\frac{\alpha}{2}\right]\right] }
\end{aligned}
$$

The total profit per unit time is calculated as follows:

$$
T P_{3}(p, T)=\frac{S R+I E-o-I P-H C-D C-P C}{T}
$$

Therefore,

$$
\begin{aligned}
& T P_{3}(p, T)=[1 / T]\left[\left[\frac{p(a-b p)\left(e^{\lambda T}-1\right)}{\lambda}\right]\left[1-\frac{\alpha}{2}\right]+\right. \\
& {\left[p I _ { e } \left[\left[\frac{(a-b p)(M-T)\left(e^{\lambda T}-1\right)}{\lambda}\right]\left[1-\frac{\alpha}{2}\right]+\right.\right.} \\
& \left.\left[\frac{(a-b p)\left(e^{\lambda T}-T \lambda-1\right)}{\lambda^{2}}\right]\left[1-\frac{\alpha}{2}\right]\right]-o-
\end{aligned}
$$

$$
\begin{aligned}
& h\left[Q t_{1}+\left[\frac{(\alpha-1)(a-b p)\left(e^{\left.\lambda t_{1}-\lambda t_{1}-1\right)}\right.}{\lambda^{2}}\right]-\right. \\
& {\left[\frac{(a-b p)(\alpha-1)}{\theta \lambda(\theta+\lambda)}\right]\left[\theta e^{\lambda t_{1}}-(\theta+\lambda) e^{\lambda T}+\right.} \\
& \left.\left.\lambda e^{(\lambda+\theta) T} e^{-\theta t_{1}}\right]\right]- \\
& {\left[\frac{c(a-b p)(\alpha-1)\left[(\theta+\lambda) e^{\lambda T}-\theta e^{\lambda t_{1}}-\lambda e^{(\lambda+\theta) T} e^{-\theta t_{1}}\right]}{[\lambda(\lambda+\theta)]}\right]-} \\
& c\left[\left[\frac{(a-b p)(\alpha-1) e^{-\theta t_{1}}}{(\lambda+\theta)}\left[e^{(\lambda+\theta) t_{1}}-e^{(\lambda+\theta) T}\right]\right]-\right. \\
& \left.\left.\left[\frac{(\alpha-1)(a-b p)\left(e^{\left.\lambda t_{1}-1\right)}\right.}{\lambda}\right]\right]\right]
\end{aligned}
$$

\section{Numerical example}

A numerical example has been presented to illustrate the proposed inventory model. The main objective is to determine the optimal price $p^{*}$ and optimal cycle length $T^{*}$ which maximizes total profit per unit time earned by the retailer. The values of different parameters are as follows:

$a=200 ; b=4 ; t_{1}=\frac{1}{12}$ year; $I_{e}=10 \% /$ year; $I_{p}=$ $15 \% /$ year; $M=0.01$ year; $\alpha=0.1 ; \theta=0.08 ; c=$ $\$ 20 /$ unit; $o=\$ 200 /$ order; $\lambda=-0.98 ; h=\$ 1 /$ unit/year.

Since $M<t_{1}$, it falls into the category of subcase 6.1 . Hence, the following results are obtained:

$p^{*}=35.5357 ; T^{*}=0.765714 ; Q^{*}=28.6351 ;$ $T P_{1}{ }^{*}=300.394$.

It can be observed from Figure 2 that the profit function plot is concave in nature.

\section{Sensitivity analysis}

Based on the above numerical example, a sensitivity analysis is executed to understand the influence of overestimation or underestimation of input parameters on the optimal values of $p, T, Q$, and total profit gained per unit time. The values of input parameters are changed from $-30 \%$ to $30 \%$ to carry out the process. The results are determined by keeping the other parameters constant and transforming one parameter at a time. The following results are obtained from Table 2.

(i) With the increment in the value of $I_{e}$, the total profit gained per unit time is observed to increase. As $I_{p}$ increases, decrement in the total profit earned per unit time is observed. With the increment in trade credit time $M$, profit earned per unit time increases since now the retailer has the opportunity to settle its payment after a longer period of time. With the increase in trade credit time, the 
retailer has the opportunity to sell most of its stock and needs to pay interest on a smaller number of left items.

(ii) With the increase in the purchasing price $c$, optimal cycle length $T$ and optimal price $p$ increases whereas total profit per unit time decreases. It is obvious that if the retailer buys goods at a higher price and if sells them at a lower price, he will incur loss. Since, demand is dependent upon selling price $p$, with the increase in selling price, demand decreases. Hence, cycle length increases and total profit per unit time decreases.

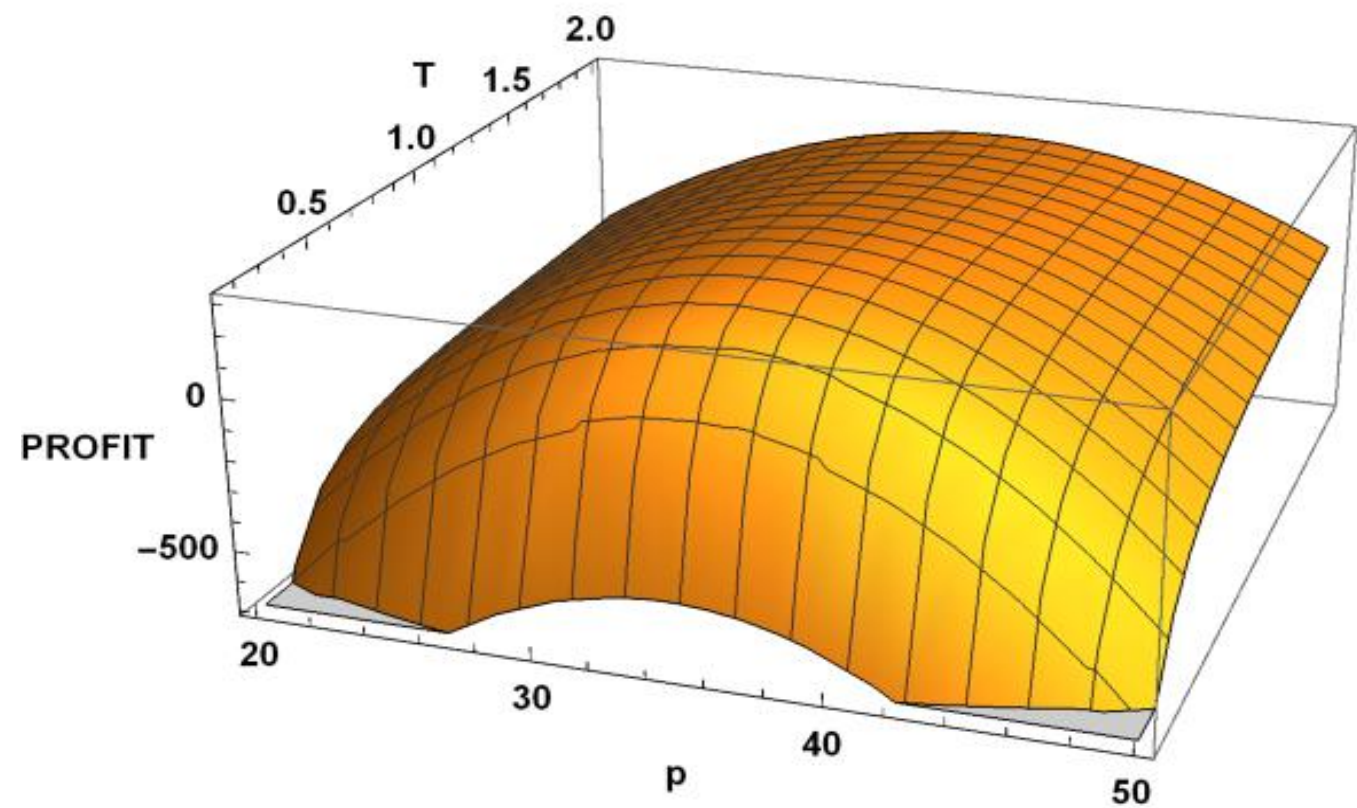

Figure 2. A three-dimensional plot showing the total profit function for the above numerical example

Table 2. Sensitivity analysis with respect to input parameters

\begin{tabular}{|c|c|c|c|c|c|}
\hline \multirow[t]{2}{*}{ Parameters } & \multirow{2}{*}{$\begin{array}{l}\% \text { Change in } \\
\text { parameters }\end{array}$} & \multicolumn{4}{|c|}{ Change in optimal values } \\
\hline & & $T P^{*}$ & $p^{*}$ & $T^{*}$ & $Q^{*}$ \\
\hline \multirow[t]{6}{*}{$I_{e}$} & -30 & 300.391 & 35.5357 & 0.765714 & 28.6351 \\
\hline & -20 & 300.392 & 35.5357 & 0.765714 & 28.6351 \\
\hline & -10 & 300.393 & 35.5357 & 0.765714 & 28.6351 \\
\hline & 10 & 300.396 & 35.5357 & 0.765714 & 28.6351 \\
\hline & 20 & 300.397 & 35.5357 & 0.765714 & 28.6351 \\
\hline & 30 & 300.398 & 35.5357 & 0.765714 & 28.6351 \\
\hline \multirow[t]{6}{*}{$I_{p}$} & -30 & 311.323 & 35.5357 & 0.765714 & 28.6351 \\
\hline & -20 & 307.68 & 35.5357 & 0.765714 & 28.6351 \\
\hline & -10 & 304.037 & 35.5357 & 0.765714 & 28.6351 \\
\hline & 10 & 296.752 & 35.5357 & 0.765714 & 28.6351 \\
\hline & 20 & 293.109 & 35.5357 & 0.765714 & 28.6351 \\
\hline & 30 & 289.466 & 35.5357 & 0.765714 & 28.6351 \\
\hline \multirow[t]{6}{*}{$M$} & -30 & 300.057 & 35.5357 & 0.765714 & 28.6351 \\
\hline & -20 & 300.169 & 35.5357 & 0.765714 & 28.6351 \\
\hline & -10 & 300.282 & 35.5357 & 0.765714 & 28.6351 \\
\hline & 10 & 300.507 & 35.5357 & 0.765714 & 28.6351 \\
\hline & 20 & 300.62 & 35.5357 & 0.765714 & 28.6351 \\
\hline & 30 & 300.733 & 35.5357 & 0.765714 & 28.6351 \\
\hline \multirow[t]{6}{*}{$c$} & -30 & 574.261 & 32.3214 & 0.628571 & 30.366 \\
\hline & -20 & 475.682 & 33.3929 & 0.662857 & 29.6643 \\
\hline & -10 & 384.27 & 34.4643 & 0.731429 & 29.7851 \\
\hline & 10 & 223.775 & 36.6071 & 0.8 & 27.3258 \\
\hline & 20 & 154.945 & 37.6786 & 0.902857 & 27.2466 \\
\hline & 30 & 93.9067 & 38.75 & 0.971429 & 26.0645 \\
\hline
\end{tabular}


Table 2. Cont.

\begin{tabular}{|c|c|c|c|c|c|}
\hline \multirow[t]{2}{*}{ Parameters } & \multirow{2}{*}{$\begin{array}{l}\% \text { Change in } \\
\text { parameters }\end{array}$} & \multicolumn{4}{|c|}{ Change in optimal values } \\
\hline & & $T P^{*}$ & $p^{*}$ & $T^{*}$ & $Q^{*}$ \\
\hline \multirow[t]{6}{*}{$o$} & -30 & 389.085 & 35.5357 & 0.594286 & 23.822 \\
\hline & -20 & 357.024 & 35.5357 & 0.662857 & 25.8368 \\
\hline & -10 & 327.436 & 35.5357 & 0.731429 & 27.731 \\
\hline & 10 & 274.965 & 35.5357 & 0.8 & 29.5118 \\
\hline & 20 & 251.321 & 35.5357 & 0.868571 & 31.1861 \\
\hline & 30 & 229.037 & 35.5357 & 0.937143 & 32.7601 \\
\hline \multirow[t]{6}{*}{$h$} & -30 & 304.149 & 35.5357 & 0.765714 & 28.6351 \\
\hline & -20 & 302.897 & 35.5357 & 0.765714 & 28.6351 \\
\hline & -10 & 301.646 & 35.5357 & 0.765714 & 28.6351 \\
\hline & 10 & 299.143 & 35.5357 & 0.765714 & 28.6351 \\
\hline & 20 & 297.892 & 35.5357 & 0.765714 & 28.6351 \\
\hline & 30 & 296.64 & 35.5357 & 0.765714 & 28.6351 \\
\hline \multirow[t]{6}{*}{$a$} & -30 & -45.9395 & 29.1071 & 2 & 19.5502 \\
\hline & -20 & 15.4218 & 31.25 & 1.48571 & 25.6462 \\
\hline & -10 & 131.364 & 33.3929 & 1.00571 & 27.4763 \\
\hline & 10 & 519.239 & 37.6786 & 0.628571 & 29.7525 \\
\hline & 20 & 786.702 & 40.3571 & 0.56 & 30.9182 \\
\hline & 30 & 1102.86 & 42.5 & 0.491429 & 31.9772 \\
\hline \multirow[t]{6}{*}{$b$} & -30 & 975.374 & 45.7143 & 0.491429 & 28.8302 \\
\hline & -20 & 677.305 & 41.4286 & 0.56 & 26.5333 \\
\hline & -10 & 460.794 & 38.2143 & 0.662857 & 27.8781 \\
\hline & 10 & 180.989 & 33.3929 & 0.868571 & 28.6064 \\
\hline & 20 & 92.5807 & 31.7857 & 1.04 & 28.6474 \\
\hline & 30 & 28.5492 & 30.1786 & 1.28 & 29.2753 \\
\hline \multirow[t]{6}{*}{$\theta$} & -30 & 310.018 & 35.5357 & 0.765714 & 28.4561 \\
\hline & -20 & 306.821 & 35.5357 & 0.765714 & 28.5155 \\
\hline & -10 & 303.613 & 35.5357 & 0.765714 & 28.5752 \\
\hline & 10 & 297.165 & 35.5357 & 0.765714 & 28.6952 \\
\hline & 20 & 293.924 & 35.5357 & 0.765714 & 28.7555 \\
\hline & 30 & 290.741 & 35.5357 & 0.731429 & 27.8973 \\
\hline \multirow[t]{6}{*}{$\lambda$} & -30 & 356.155 & 35.5357 & 0.8 & 32.806 \\
\hline & -20 & 336.532 & 35.5357 & 0.8 & 31.6531 \\
\hline & -10 & 318.01 & 35.5357 & 0.765714 & 29.6094 \\
\hline & 10 & 283.553 & 35.5357 & 0.765714 & 27.7056 \\
\hline & 20 & 267.562 & 35.5357 & 0.731429 & 26.0377 \\
\hline & 30 & 252.308 & 35.5357 & 0.731429 & 25.2459 \\
\hline \multirow[t]{6}{*}{$\alpha$} & -30 & 295.011 & 35.5357 & 0.765714 & 29.5896 \\
\hline & -20 & 296.806 & 35.5357 & 0.765714 & 29.2715 \\
\hline & -10 & 298.6 & 35.5357 & 0.765714 & 28.9533 \\
\hline & 10 & 302.189 & 35.5357 & 0.765714 & 28.317 \\
\hline & 20 & 303.983 & 35.5357 & 0.765714 & 27.9988 \\
\hline & 30 & 305.778 & 35.5357 & 0.765714 & 27.6806 \\
\hline \multirow[t]{6}{*}{$t_{1}$} & -30 & 297.756 & 35.5357 & 0.765714 & 28.6853 \\
\hline & -20 & 298.649 & 35.5357 & 0.765714 & 28.6683 \\
\hline & -10 & 299.529 & 35.5357 & 0.765714 & 28.6516 \\
\hline & 10 & 301.246 & 35.5357 & 0.765714 & 28.6189 \\
\hline & 20 & 302.084 & 35.5357 & 0.765714 & 28.6031 \\
\hline & 30 & 302.908 & 35.5357 & 0.765714 & 28.5874 \\
\hline
\end{tabular}

(iii) As ordering cost $o$ increases, simultaneously replenishment cycle length $T$ and order quantity $Q$ start increasing. It is to be observed that the total profit per unit time decreases since cycle length is increasing. With the increment in holding cost $h$, total profit per unit time decreases. (iv) With the increase in the value of $a$, it is observed that optimal values of $p, Q$, and total profit per unit time increases whereas the optimal replenishment cycle $T$ decreases. It can be seen that with the increment in the value of $b$, optimal price $p$ and total profit per unit time decreases whereas replenishment cycle length $T$ increases. 
As the value of $\theta$ i.e., deterioration rate increases, a decrease in total profit per unit time earned by the retailer is detected. With the increase in the deterioration rate, items get deteriorated faster, so total profit earned per unit time decreases

(vi) It can be seen that with the increase in the value of $\lambda$, order quantity $Q$, cycle length $T$ and total profit earned per unit time decreases. With the increment in the value of $\alpha$, optimal order quantity $Q$ decreases whereas an increment in total profit earned per unit time is observed. As the number of returns are increasing, some are resold to satisfy demand, so order quantity $Q$ decreases and total profit increases. With the increase in $t_{1}$, the time at which deterioration starts, optimal order quantity $Q$ decreases whereas total profit earned per unit time increases.

\section{Conclusion}

An inventory model with a single item is developed for a single period under both return policy and trade credit policy. If we have a glance at the literature, plenty of works on inventory model considering deteriorating items have been done. Lot of work has also been done on inventory model with trade credit policy. Some researchers have also formulated inventory models considering resalable returns for deteriorating items. Recent research recommends to follow a restrictive return policy. Nowadays, many companies offer return policies to their customers to attract them. Seller often offers buyer trade credit terms which is beneficial for the business of both of them. Till now, according to the best of author knowledge, no research work has been done which jointly studies the impact of both trade credit policy and return policy. In this paper, an attempt has been made to fill this research gap. The analysis of the impact of these two aforementioned policies in the supply chain is the main contribution of this research work. In the proposed model for deteriorating items, demand is considered to be a function of selling price and time. Retailer is offered a full trade credit period by the manufacturer whereas customers are offered with a return policy by the retailer. The returns are allowed at any time in a period. The returned product can be resold at the same selling price. Customers are not fully reimbursed for the returned product in the inventory model. Number of returns is a function of demand.

The following guidelines are recommended for future research directions: it can be extended to partial trade credit policy, a full refund of the initial amount or returning a fraction of the initial amount, an extension on multi-item and multi-period.

\section{Acknowledgement}

The authors are thankful to the anonymous reviewers and the editor for their constructive comments and suggestions. This paper has been revised in light of their suggestions and comments.

\section{References}

[1] Bechwati, N.N., Siegal, W.S. (2005). The impact of the pre-choice process on product returns. $J$. Market. Res., 42(3), 358-367.

[2] Gentry, C.R. (1999). Reducing cost of returns. Chain store Age, 75, 124-126.

[3] Mostard, J., Teunter, R., Koster, R.D. (2005). The distribution-free newsboy problem with resalable returns. International Journal of Production Economics, 97, 329-342.

[4] Palmquist, M. (2015). The unexpected benefits of product returns. Available from 〈http://www.strategy-business.com/blog/TheUnexpected-Benefits-of-Product-Returns?gko=b80 8b). Accessed 18 november 2020.

[5] Boyd, E., Bahn, K.D. (2014). Information and its impact on consumer's reaction to restrictive return policies. Journal of Retailing and Consumer Services, 21, 415-423.

[6] Harris, L.C. (2010). Fraudulent consumer returns: exploiting retailer's return policies. Eur. J. Marketing, 44(6), 730-747.

[7] Harris, L.C. (2008). Fraudulent return proclivity: an empirical analysis. J. Retailing, 84(4), 461-476.

[8] Yao, D.Q., Yue, X., Wang, X., Liu, J.J. (2005). The impact of information sharing on a returns policy with the addition of a direct channel. International Journal of Production Economics, 97(2), 196-209.

[9] Petersen, J.A., Kumar, V. (2009). Are product returns a necessary evil? Antecedents and consequences. J. Market., 73(3), 35-51.

[10] Janakiraman, N., Syrdal, H.A., Freling, R. (2016). The effect of return policy leniency on consumer purchase and return decisions: a meta-analytic review. J. Retail., 92(2), 226-235.

[11] Khouja, M., Ajjan, H., Liu, X. (2019). The effect of return and price adjustment policies on a retailer's performance. European Journal of Operations Research, 276, 466-482.

[12] Wang, K.H., Tung, C.T., \& Lee, Y.J. (2010). EOQ model for deteriorating items with resalable returns. Journal of information and Optimization Sciences, 31(1), 189-204.

[13] Ghoreishi, M., Mirzazadeh, A., \& Weber, G.W. (2014). Optimal pricing and ordering policy for non-instantaneous deteriorating items under inflation and customer returns. Optimization, 63(12), 1785-1804.

[14] Al-Salamah, M., Alsawafy, O. (2011). Economic order quantity for items with two types of imperfect 
quality. An International Journal of Optimization and Control: Theories and Applications, 2(1), 7382.

[15] Ekren, B.Y., Arslan, B. (2020). Simulation based lateral transshipment policy optimization for s, S inventory control problem in a single-echelon supply chain network. An International Journal of Optimization and Control: Theories and Applications, 10(1), 9-16.

[16] Gurkan, M.E., Tunc, H. (2021). A fix-and-optimize heuristic for the capacitated multi-item stochastic lot-sizing problem. An International Journal of Optimization and Control: Theories and Applications, 11(1), 41-51.

[17] Uddin, F.M., Sano, K. (2012). Coordination and optimization: the integrated supply chain analysis with non-linear price-sensitive demand. An International Journal of Optimization and Control: Theories and Applications, 2(1), 83-94.

[18] Mahata, G.C. (2012). An EPQ-based inventory model for exponentially deteriorating items under retailer partial trade credit policy in supply chain. Expert Syst. Appl., 39(3), 3537-3550.

[19] Min, J., Zhou, Y. W., Liu, G. Q., \& Wang, S. D. (2012). An EPQ model for deteriorating items with inventory-level-dependent demand and permissible delay in payments. International Journal of Systems Science, 43(6), 1039-1053.
Mamta Kumari has obtained her B.Sc. degree from Banaras Hindu University. She has obtained her M.Sc. degree from National Institute of Technology Durgapur. She is currently carrying out her doctoral research at National Institute of Technology Silchar. Her research area includes Operations Research, Inventory Management and Supply Chain Management.

(iD) http://orcid.org/0000-0002-7308-7405

Pijus Kanti De has obtained his M.Sc. and B.T. degrees from the University of Kalyani and received his $M$. Phil and Ph. D. degrees in Applied Mathematics from Indian School of Mines, Dhanbad (presently IIT Dhanbad). Presently he is employed as an Associate Professor in Mathematics in the National Institute of Technology Silchar. Before joining to NIT Silchar, Dr. De was employed in many other institutions like C-MMACS, National Aerospace Laboratories Bangalore, KIET Ghaziabad, Delhi College of Engineering (presently Delhi Technological University) and Banasthali University as a Senior Research Fellow, Lecturer, Sr Lecturer, Reader and Associate Professor. Dr. De has supervised several thesis at doctoral and master's level. Also Dr. De has adjudicated several doctoral thesis from different Universities. He has published many research articles and written a book, 'Computer Based Numerical Methods and Statistical Techniques'. His research areas include Operations Research, Optimization Techniques, Fuzzy Logic \& Belief Theory, Fuzzy Optimization, Fuzzy Mathematics, Mathematical Modelling, Elasto-Dynamics and Numerical Methods.

iD http://orcid.org/0000-0003-3361-9371

An International Journal of Optimization and Control: Theories \& Applications (http://ijocta.balikesir.edu.tr)

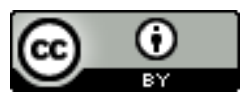

This work is licensed under a Creative Commons Attribution 4.0 International License. The authors retain ownership of the copyright for their article, but they allow anyone to download, reuse, reprint, modify, distribute, and/or copy articles in IJOCTA, so long as the original authors and source are credited. To see the complete license contents, please visit http://creativecommons.org/licenses/by/4.0/. 